\title{
A Cross-Sectional Study on Tobacco Consumption Pattern among Auto Rickshaw Drivers in Jaipur City, Rajasthan
}

\author{
Dr. Sudhir Rewar, Dr. Neha Poonia, Navneet Kumar Singh
}

\begin{abstract}
Introduction - Tobacco use is a major preventable cause of premature death and diseases, currently leading to five million deaths worldwide which are expected to raise over eight million deaths worldwide by 2030. Globally, this group of the population is under constant pressure and accounts for the workforce of the country so through this study we want to assess the prevalence of tobacco consumption and the associated factors involve in its consumption. Objective - The broad objective of study was "To assess the prevalence of tobacco consumption pattern among the auto rickshaw drivers and to study associated factors involve in tobacco use as well as associated risk factors of tobacco use in Jaipur, Rajasthan". Methodology - A cross sectional, descriptive study was done on auto rickshaw drivers of Jaipur city, Rajasthan. Study was done during April- May 2013 with a sample size of 94 with $95 \%$ of confidence level and 10\% confidence interval. Results Study finding shows that Prevalence among auto rickshaw drivers for consumption of tobacco products was very high (87\%). Auto rickshaw drivers were mostly used tobacco in the form of Gutkha (72\%) and bidi (40\%) in comparison to other products. It also shows that they use cheap tobacco products. Most of the auto rickshaw drivers start using tobacco products in age less than 18 years (80\%) and associated factors for tobacco use are due to friends and their influence (78\%). Awareness level among auto rickshaw driver was high (70\%) but still uses tobacco products because of its addiction (66\%). In the opinion of auto rickshaw drivers increase in tax may reduce it consumption and the majority of drivers (70\%) think that tobacco must be banned in Rajasthan. Conclusion - Prevalence among auto rickshaw drivers of consumption of tobacco products was very high. Auto rickshaw drivers were mostly used tobacco in the form of Gutkha (smokeless) and bidi (smoke) in comparison to other products.
\end{abstract}

Key words: Tobacco, Awareness, Prevalence, Addiction.

\section{Introduction}

Tobacco use is a major preventable cause of premature death and diseases, currently leading to five million deaths worldwide which are expected to raise over eight million deaths worldwide by 2030. Globally, cigarette smoking is the dominant form of tobacco use. The prevalence of tobacco consumption is reported by the World Health Organization (WHO), which focuses on smoking not on smokeless chewing of tobacco. India is the second largest consumer of tobacco in the world. ${ }^{(1)}$ The prevalence of tobacco use among adults (15 years and above) is $35 \%$ and the prevalence of overall tobacco use is $48 \%$ among males. ${ }^{(2)}$ Each year a great amount of money are being wasted in smoking, although it is quite obvious that smoking habit is dangerous and injurious to heath but still a large number of people especially teenagers, lower income group worker \& transport worker are attracting and getting involved in smoking habit day by day. Some reasons of this addiction are obvious such as the influence of friends or community member or to reduce work stress. This study done to assess the prevalence of tobacco consumption and the associated factors involve in its consumption. As this group of the population is under constant pressure and account for the workforce of the country. So through this study we could be able to know

- The reasons of consumption

- Amount of consumption

- Awareness of ill effect of tobacco consumption

- Out of Pocket expenditure

Rahaman M et al. - Result showed overall prevalence of smoking was $75.9 \%$. The study concluded that the prevalence of smoking among rickshaw pullers is very high compared to that of the general population. ${ }^{(3)}$ HelleSoll-Johanning et al. Mean age of smoking initiation was $15.4 \mathrm{yrs}$. The current prevalence rate of using tobacco products was $354(41.6 \%)$. Health problem were the commonest profile of the problem $(39.6 \%)$ associated with nicotine dependence. ${ }^{(4)}$ S SChaudhary et al. the study shows that $40.20 \%$ subjects had a habit of tobacco chewing; while $35.14 \%$ were smokers and $34.12 \%$ were alcohol consumers. Only $16.89 \%$ subjects were engaged in regular physical exercise. $27.36 \%$ subjects had 'moderate' or 'severe' self reported stress. 
$14.86 \%$ subjects were overweight and another $3.38 \%$ had obesity. $37.16 \%$ subjects had pre-hypertension; while $104(35.14 \%)$ subjects had hypertension. ${ }^{(5)}$

In this study we focus "To assess the prevalence of tobacco consumption pattern among the auto rickshaw drivers and to study associated factors involve in tobacco use as well as associated risk factors of tobacco use in Jaipur, Rajasthan"

\section{Methodology}

Study Design: Cross- sectional, descriptive done at Study Area- Jaipur city. Study population: Auto Rickshaw Drivers, Sampling and Sampling Design: The present cross sectional study was conducted out in Jaipur, Rajasthan, During April - May 2013. The Auto Rickshaw Drivers, a three wheeler non-motorized vehicle, were selected as study participants. Sample size - the study included 94 participants, selected through the $95 \%$ confidence level and $10 \%$ confidence interval out of 10400 Auto Rickshaw Drivers which are registered at RTO, Jaipur. Type of sampling - Simple random samplings. Data Collection Tools and Techniques: - A questionnaire has been developed, pre-tested and modified before administering it the selected participants. Questionnaire consisted of the various items which can assess the frequency of consumption, Age of initiation, the amount of consumption, mental stress, economic factors, any past history of disease and most importantly the awareness towards oral cancer. Participants were explained in detail regarding the purpose of the study; informed consent was obtained and questionnaires were distributed. The necessary steps were taken to maintain anonymity. Ethical clearance for the study was obtained from institutional ethical committee. The information thus obtained from the above questionnaire was entered in the SPSS 19 version and Descriptive study is carried out to analyse results thus obtained. The information obtained was presented in terms of mean, 95\% Confidence Intervals (CI) and percentages.

\section{Results}

In the present study out of 92 auto rickshaw drivers, 7 (7.4\%) were aged below 21 years, $44(46.8 \%)$ were in the age group of 21-35 years, $29(30.9 \%)$ were in the age group of 35-50 years and $14(14.9 \%)$ were aged more than 50 years. If we distribute population according to their educational qualification $24(25.5 \%)$ were illiterate, 39 drivers (41.5\%) were able to read and write, 11 drivers (11.7\%) had studied up to $10^{\text {th }}$ class, 5 $(5.3 \%)$ had studied up to $12^{\text {th }}$ class, $10(10.6 \%)$ had studied up to bachelor degrees and $5(5.3 \%)$ had studied up to post graduation level. If we divide study participants on the basis of their avg monthly income, more than one half participants have salary range more than Rs. 10000. In the present study out of 94 auto rickshaw drivers, 82 $(87.2 \%)$ were using tobacco products in some of its forms and $12(12.8 \%)$ were never used tobacco products. The above mentioned figures clearly reveal that there is a high prevalence among auto rickshaw drivers for consumption of tobacco products. Use of tobacco products is shown in figure1.

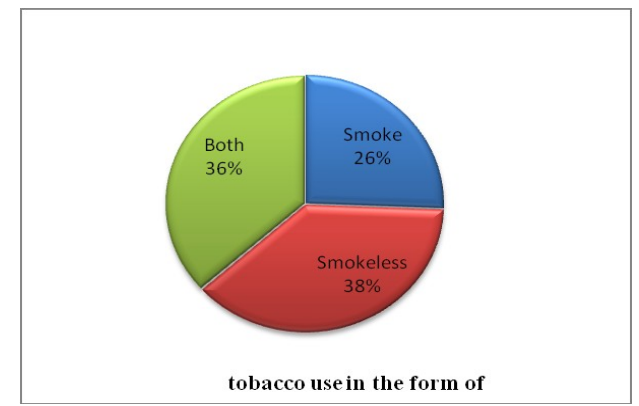

Distribution of participants according to various types of tobacco use is shown in table1.

\begin{tabular}{|l|l|l|}
\hline S.No. & Product & $\mathrm{N}=82$ \\
\hline 1 & Gutkha & 59 \\
\hline 2 & Khaini & 8 \\
\hline 3 & Cigarette & 27 \\
\hline 4 & Bidi & 33 \\
\hline 5 & Hukka & 2 \\
\hline 6 & Chutta & 0 \\
\hline 7. & snuff & 1 \\
\hline
\end{tabular}


More than $70 \%$ drivers use gutkha as first preference after that they use bidi \& cigarette form of tobacco. Distribution of study participants according to their age of initiation are shown in figure2.

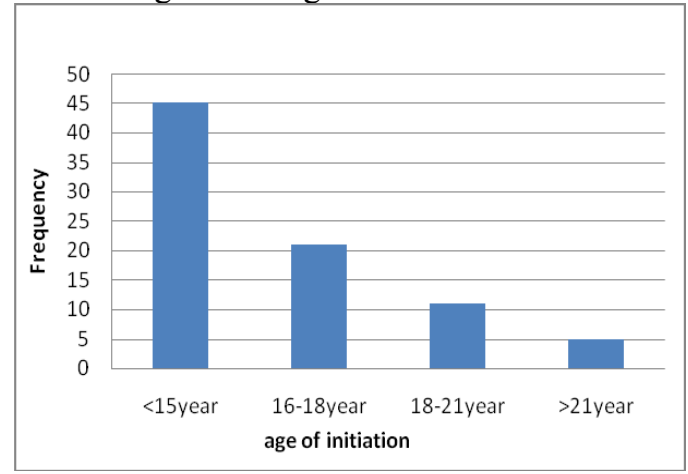

Around 55\% drivers start using tobacco products below 15 years of age. Reasons for start using of tobacco products was $64 \%$ of tobacco users started using tobacco products because of peer pressure, $24 \%$ were said that they want to experimentally use tobacco products, $8 \%$ use for style purpose and $5 \%$ start because they feel loneliness. Daily spending on using tobacco products, $11(13.40 \%)$ were spending less than Rs.10 per day on tobacco products, $41(50 \%)$ were spending Rs. $10-50$ per day, 24 (29.30\%) were spending Rs. $50-100$ per day and $6(7.30 \%)$ were spending more than Rs. 100 per day on tobacco products. Distribution of study participants according to reasons for continued use of tobacco products shown in Table2.

\begin{tabular}{|l|l|l|}
\hline S.No & Reasons & Frequency \\
\hline 1 & To reduce stress & 19 \\
\hline 2 & To be awake & 17 \\
\hline 3 & To remove nervousness & 24 \\
\hline 4 & & \\
\hline 5 & For fun & 16 \\
\hline 6 & No reasons & 42 \\
\hline
\end{tabular}

$66(70 \%)$ were aware about harmful effect of tobacco use, $21(22 \%)$ were not aware about any harmful effect of tobacco use and $7(8 \%)$ were not sure about any harmful effects of tobacco use.

But still majority of drivers were using tobacco products because of its addiction $(60 \%)$. More than one half of study population either have symptoms/ suffering from diseases. Distribution of study participants according to presence of different diseases in participants with use tobacco (percentage distribution) are shown in figure3.

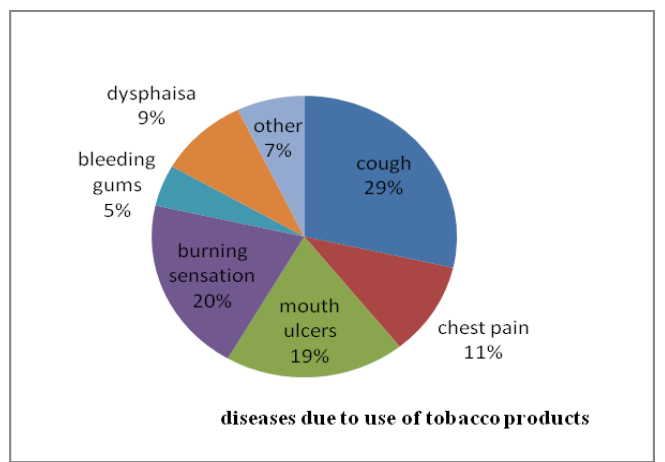

Only one tenth of total study population were able to quit his habit of using tobacco products because of encountered health problem, realised the harmful effect of tobacco, inspired by anti tobacco program and for the sake of family members. $45(48 \%)$ were saying that increase price of tobacco products reduces its consumption, $44(47 \%)$ were saying that increases price of tobacco doesn't effect on its consumption and $5(5 \%)$ were not sure about the price increase and its consumption relation. 


\section{Discussion:}

Study finding indicates that the majority of the auto rickshaw drivers are tobacco users (87\%). In the current study majority of the auto rickshaw driver were present in the age group of 21-35 years. More than $60 \%$ of total populations of the auto rickshaw driver were either illiterate or only able to read and write. In the current study majority of respondents have salary range more than Rs. 10000 . Around $50 \%$ of their family members were also in use of tobacco products. The majority of the auto rickshaw drivers daily use tobacco products while a very less number of drivers use occasionally for the purpose of fun.

Findings show that in current tobacco users; gutkha use is high in the respondents. Further results clearly points out the indigenous smoking product such as bidi which is the second most preferable choice of tobacco. In comparison to the smoking products, use of smokeless tobacco is much more common in the auto rickshaw drivers. Furthermore; it has been found that education plays its major role; as maximum users were uneducated or non-educated but they have fair knowledge about the harmful effect of tobacco use on health. In fact they understood well about the cancer related health problems due to tobacco use. The data suggest that associated factors for tobacco use are due to friends and their influence; users started this habit of tobacco in early adolescent age, i.e. Under 15 years.

The amount of consumption is very high i.e. 10-15 cigarette/bidi/gutkha in a day. Around 50\% auto rickshaw driver spend 50rs. per day on tobacco products. Reason behind to continue use of tobacco products are to reduce stress, to stay energetic during their work rather than lethargy but majority of them are into tobacco use with no reasons. More than $85 \%$ participants say that tobacco related disease can affect them despite of that they are consuming tobacco in one or the other form. Large number of the auto rickshaw drivers suffers from tobacco related diseases like cough, mouth problems (ulcer, burning sensation). Three fourth of the users tried to quit this habit but because of addiction to tobacco use; $50 \%$ participants started again. So government should focus on the interventions targeting such group of auto rickshaw drivers so that we can motivate their peer group fellows to quit the habit of tobacco use. The present study shows that increase of the price of tobacco can affect its consumption. Around $75 \%$ auto rickshaw drivers were in the favour of tobacco ban in Rajasthan. Scientific evidence shows that graphic health warnings are the most cost effective interventions to educate people regarding tobacco. In addition to this according to GATS India many attempted to limit the use of tobacco via counselling, pharmacotherapy and drug addiction centre. Hence we should encompass our strategy and policies through this technique.

But in comparisons to the past studies worldwide none of them have covered particularly auto rickshaw drivers who are the most vulnerable group of individuals sustaining to such type of abuses. But same result has been observed in the study conducted for auto rickshaw drivers "National tobacco control program-me "teachers guide". It states that use of tobacco leads to the all major respiratory and gastric problems. Addition to this my study revealed the same result as that of GATS that Gutkha is the most commonly used form of tobacco.

Affordability of the indigenous smoking and smokeless tobacco products is an important factor that increases the access to these products in the region. The smokeless tobacco products are cheaper than the cigarettes, due to the fact that the tax imposed on such products is much less than that of cigarettes. These lower the price and increase the affordability and access to the major segment of the population.

So this is important for any national level control programme or state level polices to do a deep research on the above said issues as India is one of the most prevalent country with regards to noncommunicable diseases.

\section{Conclusion}

In conclusion we say that Prevalence among auto rickshaw drivers of consumption of tobacco products was very high. Auto rickshaw drivers were mostly used tobacco in the form of Gutkha (smokeless) and bidi (smoke) in comparison to other products. It also shows that they use cheap tobacco products. Most of the auto rickshaw drivers start using tobacco products in age less than 18 years and they start it within peer pressure, which is also a high focus area of government. Mostly they use tobacco products to reduce stress, to be awake or to remove nervousness but a large number of participants also use them without any reason. Awareness level among auto rickshaw driver was high but still uses tobacco products because of its addiction. Almost one half of the study population was suffering from tobacco related diseases like cough, ulcer on mouth, lung disorder.

\section{References-}

[1] WHO Report on the Global Tobacco Epidemic, 2009: Implementing Smoke-Free Environments. Geneva, World Health Organization, 2009/http://www.who.int/tobacco/publications/gender/en_tfi_gender_women_prevalence_tobacco_use.pdf

[2] Report on oral tobacco use and its implications in south East Asia

[3] Rahaman M, Awai AS, Fukire T, Sakamoto J. Prevalence of cigarette and bidi smoking among rickshaw pullers in Dhaka city.Preventivemedicine.[online].2007.[2007 march].Availablefrom:URL:http://www.sciencedirect.com/science/article.

[4] HelleSoll-Johanning, Elsa Bach, Jørgen H Olsen, Finn Tüchsen, Occup Environ Med 1998; 55:594-598

[5] Chaudhary, M M Nagargoje1, S S Kubde2, S C Gupta3, S K Misra/Prevalence of Cardiovascular Diseases risk factors among autorickshaw drivers/Indian Journal of Community Health Vol. 22 No. 2, Vol. 23 No. 1 July 2010-June 2011 